\title{
DETECTANDO FALHAS INCIPIENTES EM MÁQUINAS ROTATIVAS
}

\section{DETECTING INCIPIENT FAULTS IN ROTATING MACHINES}

\author{
Rui F. M. Marçal ${ }^{1}$ \\ ${ }^{1}$ Doutor em Engenharia Elétrica - UFRGS 2000, Programa de Pós- \\ Graduação em Engenharia de Produção (PPGEP), Linha de \\ Pesquisa Gestão da Manutenção, Centro Federal de Educação \\ Tecnológica do Paraná (CEFET/PR), Unidade de Ponta Grossa, \\ marcal@pg.cefetpr.br \\ Altamiro A. Susin ${ }^{2}$ \\ ${ }^{1}$ Doutor em Engenharia Elétrica - INPG França 1981, Curso de \\ Pós-Graduação em Engenharia Elétrica (CPGEE), Laboratório de \\ Processamento de Sinais (LaPSI), Universidade Federal do Rio \\ Grande do Sul (UFRGS), susin@eletro.ufrgs.br
}

Recebido para publicação em: 15/09/2004
Aceito para publicação em: $12 / 06 / 2005$

\section{RESUMO}

Este trabalho apresenta um método para detectar falha em máquinas rotativas baseado no padrão de vibração do sistema e diagnostica a condição de operação por Lógica Fuzzy. As alterações são analisadas e servem para predizerem falhas possibilitando a manutenção preditiva. Utiliza-se uma estrutura denominada de Sistema Rotativo. O sistema de aquisição dos sinais é composto por um computador pessoal e um microcontrolador (Microchip PIC16C73A). As falhas inseridas neste trabalho são desbalanceamentos. Utilizam-se diferentes massas para simularem os estados de funcionamento: normal, falha incipiente, manutenção e perigo. São consideradas a freqüência de rotação do eixo_volante e as amplitudes de vibrações inerentes a cada simulação.

Palavras-Chave: Manutenção preditiva, Falha incipiente, Análise de vibração, Lógica Fuzzy.

\section{Manutenção de equipamentos}

O processo de manutenção inclui todas as atividades técnicas e organizacionais que garantam que as máquinas e equipamentos em geral operem dentro da confiabilidade esperada. Manutenção e trabalhos de reparo que seguem determinadas diretivas básicas reduzem as chances de falhas inesperadas e conseqüentes perda de produção, tempo e gastos desnecessários. Em casos mais críticos, as falhas de um processo podem trazer prejuízos graves e, até mesmo, colocar em 
risco vidas humanas. Podem-se citar três formas de manutenção: corretiva, preventiva e preditiva. (Tabela 1) [10].

\begin{tabular}{|c|c|c|c|}
\hline & $\begin{array}{c}\text { Manutenção } \\
\text { Corretiva }\end{array}$ & $\begin{array}{c}\text { Manutenção } \\
\text { Preventiva } \\
\end{array}$ & $\begin{array}{c}\text { Manutenção } \\
\text { Preditiva }\end{array}$ \\
\hline $\begin{array}{l}\text { Estado de operação da } \\
\text { máquina }\end{array}$ & Fora de serviço & Fora de serviço & $\begin{array}{l}\text { Operando ou fora de } \\
\text { serviço }\end{array}$ \\
\hline Razão da interferência & Falha & Inspeção programada & $\begin{array}{c}\text { Controle programado } \\
\text { ou contínuo }\end{array}$ \\
\hline $\begin{array}{c}\text { Tarefas a serem executadas } \\
\text { na máquina }\end{array}$ & $\begin{array}{l}\text { Reposição de } \\
\text { componentes }\end{array}$ & $\begin{array}{c}\text { Desligamento da máquina para } \\
\text { inspeção ou reposição de } \\
\text { componentes }\end{array}$ & Monitoramento \\
\hline Objetivo da intervenção & $\begin{array}{l}\text { Retorno ao } \\
\text { trabalho }\end{array}$ & $\begin{array}{l}\text { Garantir o funcionamento por } \\
\text { um tempo }\end{array}$ & $\begin{array}{l}\text { Predizer ou detectar } \\
\text { falhas }\end{array}$ \\
\hline
\end{tabular}

Tabela 1 - Modalidades de manutenção e suas características

\subsection{Parâmetros medidos em manutenção preditiva}

Os parâmetros a serem medidos (Tabela 2) [10], devem fornecer informações que permitam a inspeção de elementos específicos da máquina ou do tipo de falha. A opção pelo parâmetro e a forma de investigação mais adequada, que retrate com maior acuidade o que se investiga, são fatores relevantes e determinantes (pequenos mecanismos podem ter alteração de vibração ao serem colocados em contato com sensores) [8]. Através dos dados conseguidos pode-se ter uma análise técnica, onde os resultados desta análise indicaram a natureza da falha; que se pode esperar; e estabelecer quais são os elementos críticos do sistema. Pode-se, ainda, montar um histórico do equipamento, que permitirá estabelecer quais são os elementos de falha mais frequientes e o tempo decorrido entre as falhas [8].

\begin{tabular}{|l|l|}
\hline \multicolumn{1}{|c|}{ Parâmetro a ser medido } & \multicolumn{1}{c|}{ Natureza da falha ou defeito a ser detectado } \\
\hline $\begin{array}{l}\text { Amplitude do } \\
\text { Deslocamento de vibração }\end{array}$ & $\begin{array}{l}\text { Desbalanceamento, falta de alinhamento, folgas, má fixação, mal } \\
\text { acoplamento, correias frouxas, eixo torto,... }\end{array}$ \\
\hline $\begin{array}{l}\text { Amplitude da } \\
\text { Velocidade da vibração }\end{array}$ & Mancais ou rolamentos defeituosos,... \\
\hline $\begin{array}{l}\text { Amplitude da } \\
\text { Aceleração da vibração }\end{array}$ & $\begin{array}{l}\text { Estado dos rolamentos, fricção excessiva entre componentes, falta de } \\
\text { lubrificação nos mancais,... }\end{array}$ \\
\hline Freqüência de vibração & $\begin{array}{l}\text { Dados complementares para monitoramento de qualquer característica } \\
\text { de vibração essencial na determinação de qualquer problema } \\
\text { detectado,... }\end{array}$ \\
\hline
\end{tabular}

Tabela 2 - Parâmetros a serem medidos em manutenção preditiva 


\section{Análise vibracional na monitoração de máquinas}

As máquinas são elementos mecânicos complexos, articulados. As peças que sofrem excitação podem oscilar e as oscilações transmitem-se pelas articulações aos demais elementos acoplados. O resultado é um complexo de freqüências que caracteriza o sistema.

Cada vez que uma peça altera suas características mecânicas por desgaste ou trinca, uma componente de freqüência do sistema será alterada. Havendo alteração no acoplamento entre as peças, altera o coeficiente de transmissão do sinal entre as peças e, em conseqüência, a forma de freqüência global do sistema.

Folgas, defeitos ou desalinhamentos de rolamentos ou mancais de máquinas rotativas refletem-se na alteração de freqüências ou no surgimento de novas freqüências. $O$ desbalanceamento do rotor é transmitido pelo rolamento.

As forças centrífugas, alternativas e de fricção atuantes nos distintos elementos de uma máquina em operação, dão origem a vibrações mecânicas proporcionais, que se manifestam nos mancais. Devido a este fato, medindo-se vibrações nos mancais pode-se detectar e determinar os esforços presentes em quaisquer componentes da máquina, determinando-se eventuais anormalidades de funcionamento. Em geral a medida de vibrações deve ser efetuada nos mancais, por ser um dos pontos válidos pelas normas em uso empregadas para avaliar o funcionamento de máquinas [10].

A premissa fundamental sobre a qual se baseia a análise de vibração como técnica aplicada à manutenção industrial é: “Cada componente ou cada tipo de deficiência mecânica de uma máquina em operação produz uma vibração de freqüência específica que em condições normais de funcionamento, alcança uma amplitude máxima determinada" [10]. Desta feita, é possível medindo-se e analisando-se a vibração, se estabelecer sua origem, identificar cada componente da máquina e o tipo de falha que a está gerando, além, de avaliar o estado mecânico do componente que a produz ou a gravidade da deficiência detectada.

A metodologia básica recomenda a:

1) Medição de freqüência para identificar a origem da vibração;

O conhecimento da freqüência permite identificar o componente da máquina ou a natureza da falha que produz a vibração

2) Medição da amplitude para avaliar a vibração e consequentemente o funcionamento normal ou anormal do sistema; 
A medição da amplitude permite avaliar por comparação com valores limites previamente estabelecido se a vibração corresponde a um funcionamento normal ou anormal e o grau de importância da falha detectada.

\subsection{Freqüiência da vibração}

Freqüência é a razão de repetição de um evento periódico, geralmente expressa em rotações (ou ciclos) por segundo, Hz; rotações por minuto, rpm; ciclos por segundos, cpm ou múltiplos da velocidade de rotação, harmônicos. Harmônicas são comumente referidas como sendo 1x a rotação, $2 x$ a rotação, $3 x$ a rotação e assim sucessivamente.

Condições tais como: instabilidade, desbalanceamento, desalinhamentos, mudanças no ajuste, desgaste e até mesmo fadiga em seus componentes, geram vibrações específicas e características. A vibração característica mais comum ocorre na frequiência de rotação da máquina. A vibração na freqüência de rotação da máquina é geralmente a componente com a maior amplitude na medida em velocidade ou deslocamento.

\subsection{Análise espectral}

O método de análise de frequiências, baseado no teorema de Fourier, estabelece que qualquer função periódica possa ser decomposta por uma série de ondas sinusoidais puras com freqüências distintas e múltiplas harmônicas da freqüência fundamental. Estas componentes constituem o espectro de freqüência da vibração. Ao se utilizar transdutores sensores (acelerômetros) para a aquisição de tal parâmetro e posterior análise, o espectro de vibração de um sistema pode ser levantado. Observando-se a amplitude dos picos em determinadas frequiências e relacionando-se as amplitudes observadas com as da freqüência fundamental do sistema (rotativo), pode-se chegar a um diagnóstico do estado de funcionamento ou alterabilidade em curso do sistema.

\subsection{Determinação da variável a ser medida}

Cada força excitadora existente em diferentes pontos da máquina gerará uma harmônica da vibração determinando certo deslocamento, certa velocidade e uma dada aceleração; a soma de todas as harmônicas para cada variável resultará uma poliharmônica, presente nos mancais.

Pode-se classificar o total das componentes harmônicas da vibração em dois grupos, delineados pelo valor da freqüência de rotação do eixo. Assim, se estabelece a divisão [10]:

1) Componentes de baixa freqüência (valores de freqüência até 5 vezes a rpm do eixo);

2) Componentes de alta frequiência (valores maiores que 5 vezes a rpm do eixo). 
Convém esclarecer que os valores acima fixados seguem uma divisão orientativa.

\section{O processo de aquisição da assinatura espectral}

\subsection{O sistema rotativo}

Utiliza-se um sistema rotativo (figura 1), composto por um motor com uma rotação nominal de 1800 rpm e um sistema de polias, cuja relação é 1.3, que aciona por correia um eixo_volante sustentado em mancais com buchas de bronze. O eixo_volante gira em 2270 rpm, medidos com a utilização de um tacômetro MICROTEST L20. Neste eixo, mantém-se na extremidade oposta à da polia um volante de $15 \mathrm{~cm}$ de diâmetro e $1012 \mathrm{~g}$ de massa.

Segundo YA'CUBSOHN, podem-se dividir as falhas em duas classes genéricas: as de baixa frequiência (ex: desbalanceamentos, desalinhamentos, etc.) e as de alta frequiência (ex: rolamentos deteriorados, falta de lubrificação, etc.). Entretanto, não basta medir a freqüência de cada um dos componentes da vibração para identificar a falha, deve-se, uma vez conhecido o valor absoluto, relacioná-lo com a velocidade de giro do eixo. Portanto, o conhecimento da velocidade de rotação do eixo ou do sistema rotativo, em questão, se torna necessário para o diagnóstico de falhas.

Tem-se que, quaisquer modificações ocorridas devido à desbalanceamento, desalinhamentos, jogo excessivo, falta de rigidez, acoplamento defeituoso, correias frouxas ou gastas, eixos deformados, desajustes, Tc, alterará a amplitude da vibração na frequiência de rotação do sistema rotativo em questão [10]. Tais falhas podem ser classificadas como uma família de falhas de baixa freqüência e aqui são nomeadas como desajustes.

Uma das dificuldades em detecção de falhas em motores é a alta dimensão física dos motores. Existem muitas variáveis que podem afetar o processo de detecção de falhas, tais como: condições de carga, efeitos de saturação, condições de operação imprevisível, ruídos de linha e efeitos de temperatura, os quais podem resultar em milhares de hipóteses pelas diferentes possibilidades de combinações destas variáveis [2].

As relações de massa dos demais elementos desbalanceadores são estabelecidas segundo um critério adotado para a classificação do diagnóstico oferecido pelo sistema especialista Fuzzy: 0,1 g => FALHA INCIPIENTE; 5,1 g $\Rightarrow$ MANUTENÇÃO; 7,2 g $\Rightarrow$ PERIGO.

Os valores acima citados são específicos para este equipamento. Cada instalação tem seus parâmetros de operação e faixas de tolerância. O método proposto tem uma fase de sintonia do sistema de supervisão, chamada de "sintonia do sistema especialista Fuzzy", na qual são estabelecidas as condições de operação. 


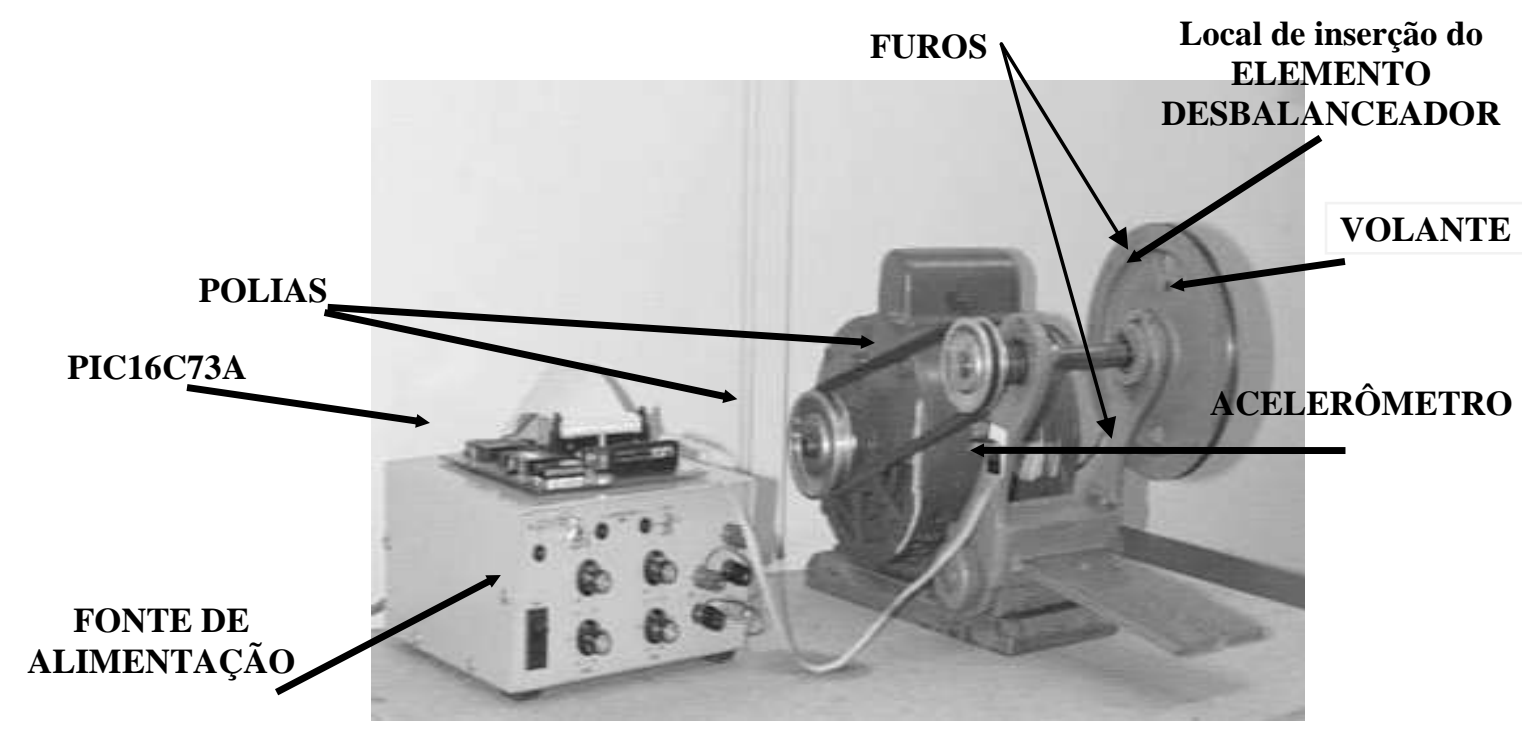

Figura 1 - O sistema rotativo

\section{2. $O$ acelerômetro empregado}

Usa-se o ADXL202, acelerômetro em chip biaxial de baixa potência da Analog Device. O ADXL202 pode medir acelerações dinâmicas (vibração) até \pm 2 g e aceleração estática (gravidade). Tem-se nas saídas (eixos x e y) do acelerômetro um sinal digital cuja informação da aceleração é o tempo de duração de um sinal "duty cycle”. As saídas podem ser lidas diretamente por um contador microprocessador não requerendo um conversor A/D.

\subsection{O sistema de aquisição}

O sistema de aquisição é composto por dois subsistemas: um computador pessoal e um microcontrolador. O computador através de um software de aplicação processa os dados de aceleração, o diagnóstico Fuzzy e permite a interface do usuário. O sistema é baseado em um microcontrolador (PIC16C73A) que faz a interface do acelerômetro e o computador. A interface entre o microcontrolador e o acelerômetro é dotada de um contador que conta o tempo do período do sinal PWM. O software que roda no microcontrolador constrói uma estrutura de dados contendo a informação de aceleração do sensor. Estes dados são enviados para o "host” do computador através da entrada serial. A aceleração é processada pelo tempo de duração do período do sinal PWM 


\subsection{As aquisições das alterações}

Para se chegar a um padrão de vibração, denominada assinatura espectral do sistema (figura 2), adquire-se 10 arquivos, os quais, uma função desenvolvida em C encontra a média. Desta forma, consideram-se as possíveis variações que possam ocorrer em um regime constante de funcionamento e até mesmo no desligar e religar o sistema para a inserção das falhas. As falhas inseridas nesta simulação são desbalanceamentos no eixo_volante através da inserção de elementos desbalanceadores.

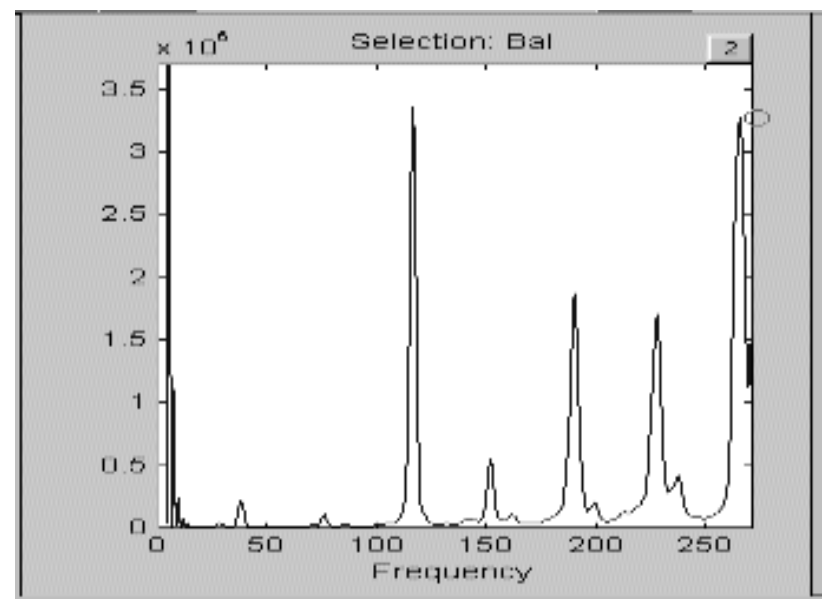

Figura 2 - Assinatura espectral do sistema rotativo

Ressalta-se que, não se consegue inferir as avarias de desbalanceamento com o sistema em funcionamento. A figura 3 traz o espectrograma das aquisições.

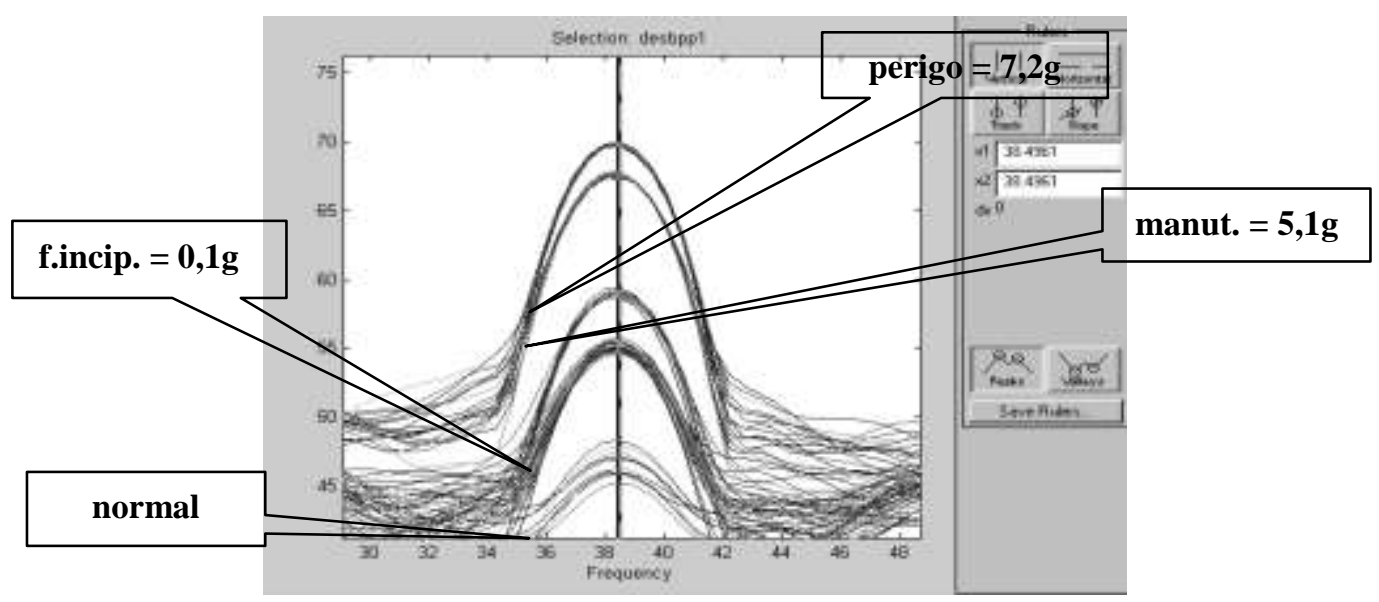

Figura 3 - Espectrograma com um grupo de 10 aquisições 
O eixo_volante possui um furo no qual se inserem elementos desbalanceadores, um de cada vez, segundo três relações estabelecidas: 1 para 10.000 (usa-se um peso de $0,1 \mathrm{~g}), 1$ para $200(5,1 \mathrm{~g}$ ) e 1 para 140 (7,2 g). Tais relações ocasionam diferentes graus de desbalanceamentos no sistema rotativo e estabelecem, segundo um critério de classificação (falha incipiente, manutenção e perigo) sugerido por especialistas, as variáveis lingüísticas empregadas no sistema Fuzzy.

\subsection{O processamento com Matlab}

O método proposto está separado por estágios e operacional em ambiente Matlab (figura 4). É possível executá-lo por passos: aquisição dos dados (*.dat), média das aquisições, determinação e plotagem da assinatura espectral, busca de valores de amplitude na freqüência de rotação do sistema rotativo, análise e diagnóstico Fuzzy. Também permite a execução de todas as etapas a partir de um comando e a seleção de tempos de aquisições, quantidade de aquisições, faixa de frequiência para análise e calibração de todo o sistema Fuzzy.

A análise no domínio frequiência é possível com a utilização do programa SPTOOL, que permite dentre outras facilidades o enjanelamento da faixa de freqüência pretendida para a análise e monitoramento. Tem-se que, qualquer modificação ocorrida devido ao desbalanceamento se fará sentir com mudanças de amplitude na freqüência de rotação do sistema rotativo em questão. Daí a análise neste ponto do espectrograma.

\section{O diagnóstico Fuzzy}

A metodologia permite a chegada a um diagnóstico do estado de funcionamento do sistema rotativo, pelo sistema Fuzzy, baseada em uma coleção de regras lógicas na forma de declarações IFTHEN. As declarações “antecedentes” são: a freqüência de rotação do eixo_volante e a amplitude da vibração nesta freqüência. A declaração "conseqüente" é o diagnóstico do estado de funcionamento do sistema (Figura 4). 


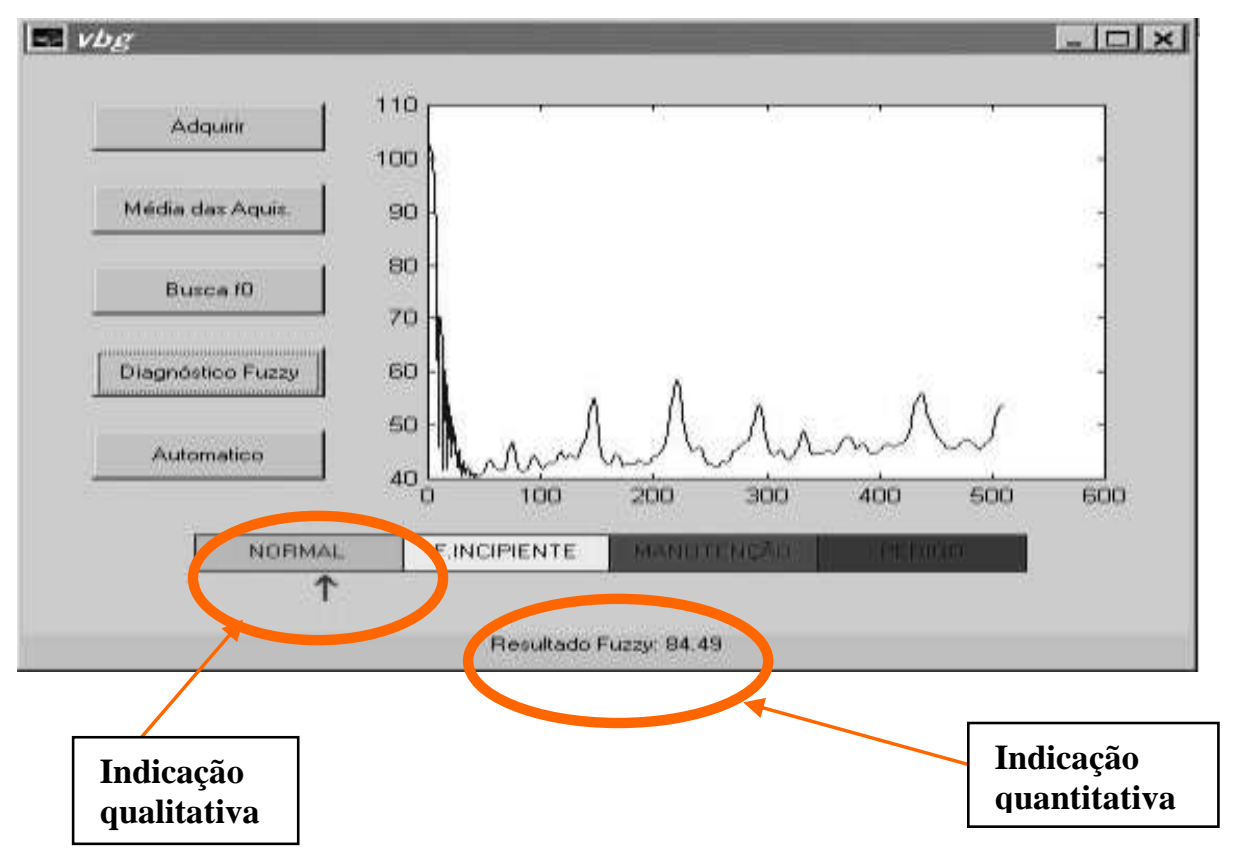

Figura 4 - O diagnóstico fuzzy

A facilidade e praticidade de alterações das regras e redefinição de valores das variáveis a serem consideradas, permitem que a metodologia se apresente como um método versátil. A calibração possibilita que o método possa atender às necessidades específicas do processo em que o sistema rotativo está inserido. Pode-se calibrá-lo levando-se em consideração a margem de atuação desejada para o sistema, a qualidade do material empregado na construção e fatores de risco e tolerância.

O diagnóstico é oferecido pelo sistema Fuzzy de modo quantitativo e qualitativo, sendo desta forma possível o acompanhamento da evolução da modificação incipiente para uma falha ou defeito de funcionamento.

\section{Conclusão}

O método pode ser aplicado a outros sistemas rotativos desde que devidamente adaptado. Esta adaptação refere-se desde a monitoração propriamente dita (o monitoramento de sistemas rotativos que apresentem freqüências de rotação diferentes do que foi usado para a comprovação) como também a faixa de atuação dos valores que identificam os estados de operação do sistema (a faixa pode ser ajustada pela sintonia do sistema especialista Fuzzy). 
Atende à proposta inicial que propõe a implementação da modalidade de manutenção preditiva, onde se pode pelo diagnóstico apresentado programar o momento certo de atuação no sistema.

\begin{abstract}
This work provides a detection method for faults in the operation of rotating machines based on a change of system vibration standard and in the operation status diagnosis by Fuzzy logic. These changes are used as parameters for predicting incipient faults, as well as their evolution in operation condition allowing predictive maintenance tasks. A mechanic structure called Rotating System has been used. The data acquisition of structure vibration has been made by a biaxial solid state accelerometer in a low-power chip. The acquisition system is composed by a personal computer and a microcontroller based front-end (Microchip PIC16C73A). The faults analyzed in this work are due to the unbalancing of axle_wheel by insertion of unbalanced elements. The Fuzzy system was calibrated to detect and diagnose the normal, incipient fault, maintenance, and danger conditions of the Rotating System.
\end{abstract}

Key words: Predictive maintenance, incipient faults, vibration analyze, Fuzzy Logic

\title{
6. Bibliografia
}

[1] BORCHARDT, I. G.; SUSIN, A. A.; BRITO, R. M. Uma taxonomia para a instrumentação. In: CINISA, 3., São Paulo. Anais... São Paulo, dez. 1995.

[2] CHOW, M. Y. Methodologies of using neural network and fuzzy logic technologies for motor incipient fault detection. Singapore : World Scientific Publishing Co. Pte. Ltd., 1997.

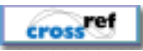

[3] DOEBELIN, E. D. Measurement systems application and design. $4^{\text {th }}$ ed. New York : McGraw Hill, 1990.

[4] GERGES, S. N. Y.; LIMA, N. N. C. Análise de vibrações para diagnóstico de falhas em engrenagens. Manutenção, pp. 18-22, jul. 1987. [Laboratório de Vibrações e Acústica, Departamento de Engenharia Mecânica da UFSC,

Florianópolis, SC].

[5] MECHEFSKE, C. K. Machine condition monitoring: part 1 - Optimum vibration signal lengths. British Journal of NDT, v. 35, n. 9, pp. 503-507, Sept. 1993.

[6] MECHEFSKE, C. K. Machine condition monitoring: part 2 - The effects of noise in the vibration signal. British Journal of NDT, v. 35, n. 10, pp. 574-579, Oct. 1993.

[7] ASSOCIAÇÃO BRASILEIRA DE NORMAS TÉCNICAS. NBR 8008 - Balanceamento de corpos rígidos rotativos. ABNT : Rio de Janeiro, 1983.

[8] RITTER, M. A. Análise vibracional aplicada ao monitoramento de máquinas rotativas. Monografia (Graduação em Engenharia Mecânica) - UFRGS, Porto Alegre, 1996.

[9] VARGA, G.; LIPOVSZKY, G.; SÓLYOMVÁRI, K. Vibration testing of machines and their maintenance. Amsterdam : Elsevier, 1990.

[10] YA'CUBSOHN, R. V. El Diagnostico de fallas por análisis vibratorio. São Paulo : Die Techik Ltda., 1983. 